\title{
Uncertainty principle for the entropy of black holes, de Sitter and Rindler spaces
}

\section{Tetsuya Hara, Keita Sakai, Shuhei Kunitomo and Daigo Kajiura}

Department of Physics, Kyoto Sangyo University, Kyoto 603-8555, Japan

email: hara@cc.kyoto-su.ac.japan

\begin{abstract}
By a simple physical consideration and uncertain principle, we derive that temperature is proportional to the surface gravity and entropy is proportional to the surface area of the black hole. We apply the same consideration to de Sitter space and estimate the temperature and entropy of the space, then we deduce that the entropy is proportional to the boundary surface area. By the same consideration, we estimate the temperature and entropy in the uniformly accelerated system (Rindler space). The cases in higher dimensions are considered.
\end{abstract}

Keywords. Uncertainty principle - black hole entropy - de Sitter space - Rindler space

\section{Introduction}

Although it has passed almost 30 years since the discussion of the thermodynamics of black hole and event horizon began, there are still many problems about the fundamental concepts. One of them is that the entropy of the black hole is proportional to its surface. The other is that the temperature of the black hole and event horizon is related to the acceleration strength. These results could be understood if heuristic assumptions are adopted. One of the key concept is the uncertainty principle. We apply it to the black hole, de Sitter space and Rindler space to derive the characteristic features about the entropy in these spaces.

\section{Black hole}

As the black hole of mass $M$ has the gravitational radius $r_{g}=2 G M / c^{2}$, there is the corresponding momentum $\Delta p=\hbar /\left(2 c_{1} r_{g}\right)$ from the uncertainty principle $\Delta p \Delta x \simeq \hbar / 2$, putting $\Delta x$ as $\Delta x \simeq c_{1} r_{g}$. If the black hole is formed by photons corresponding to this momentum, it could be speculated that the temperature of the black hole is proportional to the surface gravity $k T \simeq \Delta p c \simeq 1 /\left(4 c_{1} G M\right) \simeq M / r_{g}^{2}$ and it could be explained that the entropy of the black hole is proportional to the surface of the black hole.

If the black hole is formed by the black body radiation of temperature $T$, the volume $V$, total photon number $N$ and total entropy $S$ are given by

$$
V=\frac{M c^{2}}{\epsilon}=\frac{15}{\pi^{2}} \frac{(8 \pi G)^{4} M^{5}}{\hbar c^{7}}, \quad N=n V=\frac{30 \zeta(3)}{\pi^{4}} \frac{8 \pi G}{\hbar c} M^{2} \simeq \frac{240}{\pi^{3}} \frac{G}{\hbar c} M^{2},
$$

$S / k_{B}=s V / k_{B}=\frac{32 \pi}{3}\left(G M^{2} / \hbar c\right)$, where the following radiation density $\epsilon$, number density $n$, and entropy density $s$ of the radiation temperature $T$ are used $\left(\epsilon=\tilde{a} T^{4}\right.$, $n=\frac{2 \zeta(3)}{\pi^{2}}\left(\frac{k_{B} T}{\hbar c}\right)^{3}=0.244\left(\frac{k_{B} T}{\hbar c}\right)^{3}, s=\frac{4}{3} \tilde{a} T^{3}, \frac{n}{\frac{s}{k_{B}}}=\frac{\frac{2}{\pi^{2}} \zeta(3)}{\frac{\pi^{2}}{15} \times \frac{4}{3}}=\frac{45 \zeta(3)}{2 \pi^{4}} \simeq 0.2776$, being $\tilde{a}=\pi^{2} k_{B}^{4} /\left(15 \hbar^{3} c^{3}\right)$ and $\zeta(3)=1.202$ the radiation constant and zeta function).

Using the surface of the black hole $A=4 \pi r_{g}^{2}=16 \pi G^{2} M^{2} / c^{4}$, and taking $c_{1}=\frac{3 \pi}{4}$, the following relation is derived, $S / k_{B} \simeq c^{3} A /(4 G \hbar) \simeq A /\left(4 \ell_{p}^{2}\right)$, where $\ell_{p}=\sqrt{G \hbar / c^{3}}$ is the Planck length. 


\section{De Sitter space}

The SNe Ia and WMAP observations confirm that our universe is now accelerating. For simplicity, we consider the universe with $\Lambda$ term as de Sitter space with metric

$$
d s^{2}=-\left(1-\frac{\Lambda}{3} r^{2}\right) c^{2} d t^{2}+\left(1-\frac{\Lambda}{3} r^{2}\right)^{-1} d r^{2}+r^{2}\left(d \theta^{2}+\sin ^{2} \theta d \phi^{2}\right) .
$$

The characteristic point of this space is that there is the horizon with the radius of $\ell_{\Lambda}=\sqrt{3 / \Lambda}$ (de Sitter horizon). Applying the uncertainty principle for this length, the energy $\Delta E$ for the photon or particle is estimated as $\Delta E \simeq \Delta p c \simeq \frac{\hbar c}{2 \Delta x} \simeq k_{B} T$. Taking $\Delta x=c_{3} \ell_{\Lambda}$, the temperature $T$ is given by $k_{B} T=\frac{\hbar c}{2 c_{3} \ell_{\Lambda}} \simeq \frac{\hbar c}{2 c_{3} \sqrt{3}} \sqrt{\Lambda}$. When we put $c_{3}=\pi$ and the acceleration of the space as $a=c^{2} \sqrt{\Lambda / 3}$, it becomes the one $k_{B} T=\hbar c \sqrt{\Lambda} /(2 \pi \sqrt{3})=a \hbar /(2 \pi c)$ what Gibbons and Hawking have derived.

Because the cosmological constant is related to the vacuum energy density $\rho_{\Lambda}$ as $\rho_{\Lambda}=$ $\frac{\Lambda c^{2}}{8 \pi G}$ the number density of the particle is given by $n_{\Lambda} \frac{\Delta E}{c^{2}}=\rho_{\Lambda}$. Assuming that particle energy is given by $\Delta E=\hbar c / 2 c_{3} \ell_{\Lambda}$, the number density $n_{\Lambda}$ becomes as $n_{\Lambda}=\frac{\rho_{\Lambda} c^{2}}{\Delta E}=$ $\frac{\Lambda c^{4}}{8 \pi G} \frac{2 c_{3} \ell_{\Lambda}}{\hbar c}=\frac{3 c_{3}}{4 \pi G} \frac{c^{3}}{\hbar \ell_{\Lambda}}$. Taking the volume of the universe $V$ as $V=\frac{4}{3} \pi \ell_{\Lambda}^{3}$, the total number is given by $N_{\Lambda}=n_{\Lambda} V=\frac{3 c_{3}}{4 \pi G} \frac{c^{3}}{\hbar \ell_{\Lambda}} \frac{4}{3} \pi \ell_{\Lambda}^{3}=c_{3}\left(\frac{c^{3}}{G \hbar}\right) \ell_{\Lambda}^{2}=c_{3} \frac{\ell_{\Lambda}^{2}}{\ell_{p}^{2}}$.

If we assume the particle as Bose particle such as photon, the total entropy is proportional to the total number as $\left(N /\left(S / k_{B}\right)=0.2776\right) \frac{S}{k_{B}} \sim \frac{N}{0.2776} \sim 4 c_{3} \frac{\ell_{\Lambda}^{2}}{\ell_{p}^{2}}$. Using the area of the de Sitter horizon $A=4 \pi \ell_{\Lambda}^{2}$, it is expressed as $\frac{S}{k_{B}} \sim \frac{c_{3}}{\pi} \frac{A}{\ell_{p}^{2}}$, where the entropy is proportional to the horizon area $A$. If we take $c_{3}=\pi / 4$, it becomes $S / k_{B}=A /\left(4 \ell_{p}^{2}\right)$ which is derived by Gibbons and Hawking.

\section{Rindler space}

Unruh Effect has been said that in the uniformly accelerating coordinate (Rindler coordinate) he or she (observer) seems to be in a bath of blackbody radiation at the temperature $T$ which is related to the acceleration $\kappa(=a)$ as $k_{B} T=\hbar \kappa /(2 \pi c)$.

There is a characteristic length $\ell_{\kappa}=c^{2} / \kappa$ due to the acceleration $\kappa$. Applying the uncertainty principle to this length, the energy $\Delta E$ of the particle is given by $\Delta E \simeq$ $\Delta p c \simeq \frac{\hbar c}{2 \Delta x} \simeq \frac{\hbar c}{2 c_{4} \ell_{\kappa}} \simeq \frac{\hbar \kappa}{2 c_{4} c} \simeq k_{B} T$, where we put $\Delta x=c_{4} \ell_{\kappa}$. The relation between the temperature and the acceleration $\kappa$ is given by $k_{B} T \simeq \frac{\hbar \kappa}{2 c_{4} c}$. If we take $c_{4}=\pi$, the result is the same derived by Unruh.

In the following we consider the relation of this temperature to the entropy as $k_{B} S=$ $\frac{A}{4 \ell_{p}^{2}}$. One way of the derivation is to assume that the acceleration $\kappa$ is the gravitational acceleration by the mass $M$, which is composed of the blackbody radiation of temperature $T$. If we take the volume of the considering region as $V$, the mass is given as $M=\frac{\tilde{a} T^{4}}{c^{2}} V$. The acceleration $\kappa$ due to this mass is given by $\kappa=\frac{G M}{V^{\frac{2}{3}}}=\frac{G \tilde{a} T^{4}}{c^{2}} \frac{V}{V^{\frac{2}{3}}}=\frac{G \pi^{2}}{15} \frac{k_{B}^{4} T^{4}}{\hbar^{3} c^{3}} \frac{V^{\frac{1}{3}}}{c^{2}}$. Here we use the relations $k_{B} T=\hbar c /\left(2 c_{4} \ell_{\kappa}\right)$, the above equation becomes $\kappa=\frac{c^{2}}{\ell_{\kappa}}=$ $\frac{\pi^{2}}{15} \frac{1}{16 c_{4}^{4}} \frac{G \hbar c}{c^{2}} \frac{V^{\frac{1}{3}}}{\ell_{\kappa}^{4}}$. Then the region size is given by $V^{\frac{1}{3}}=\frac{15 \times 16 c_{4}^{4}}{\pi^{2}} \frac{\ell_{\kappa}^{3}}{\ell_{p}^{2}}$.

As the total entropy is $S=\frac{4}{3} \tilde{a} T^{3} V$, the entropy per surface $S / V^{\frac{2}{3}}$ is given by

$$
\frac{S / k_{B}}{V^{\frac{2}{3}}}=\frac{4}{3} \frac{\pi^{2}}{15} \frac{k_{B}^{3} T^{3}}{\hbar^{3} c^{3}} V^{\frac{1}{3}}=\frac{8}{3} \frac{c_{4}}{\ell_{p}^{2}} .
$$

If we take $c_{4}=\frac{3}{32}$ and $A=V^{\frac{2}{3}}$, the relation $\frac{\frac{S}{k_{B}}}{A}=\frac{1}{4 \ell_{p}^{2}}$ is derived, which means that the entropy per surface is $1 /\left(4 \ell_{p}^{2}\right)$. Finally, we derived the relation $S /\left(k_{B}\right)=A /\left(4 \ell_{p}^{2}\right)$, which is common for the black hole and de Sitter space. 